\title{
La révolution X-FEL Des lasers à rayons $X$ pour sonder la matière
}

\author{
Éric Collet $^{(1)}$ (eric.collet@univ-rennes1.fr), Marion Harmand ${ }^{(2)}$, Marie-Emmanuelle Couprie ${ }^{(3)}$ \\ et Marco Cammarata ${ }^{(1)}$ (marco.cammarata@univ-rennes1.fr) \\ (1) Institut de Physique de Rennes, UMR 6251 UR1-CNRS, Université de Rennes 1, 35042 Rennes Cedex \\ (2) Institut de Minéralogie, de Physique des Matériaux et de Cosmochimie (IMPMC), \\ UMR 7590 CNRS-Université P. et M. Curie-IRD-MNHN, 4 place Jussieu, 75004 Paris Cedex \\ (3) Synchrotron S0LEIL, Saint-Aubin, BP 48, 91192 Gif-sur-Yvette Cedex
}

Les études de la structure

de matériaux à l'échelle

atomique ou moléculaire

à l'aide d'expériences

de diffraction des rayons $X$

ont profondément changé

notre façon d'appréhender

l'origine microscopique

de leurs propriétés.

Les X-FELs (lasers X à électrons

libres) sont de nouvelles

sources de rayons $\mathrm{X}$,

dont les performances

ouvrent des perspectives

extraordinaires pour étudier

les propriétés structurales

et dynamiques de la matière.

Il est à présent possible

d'observer des mouvements

atomiques en temps réel,

d'étudier des nanocristaux

ou encore d'imager

des objets très petits comme

des virus ou des protéines.
C'est en 1895 que Wilhelm Conrad Röntgen découvre les rayons $\mathrm{X}$ et leur faculté à pénétrer la matière. La possibilité de faire diffracter ces rayonnements par les cristaux, démontrée par Max von Laue en 1912, a permis dès 1913 à William Henry Bragg et William Lawrence Bragg de révéler les premières structures cristallines comme celle du diamant. Les atomes, qui ont la faculté de diffuser les rayons $\mathrm{X}$ de longueur d'onde $\lambda$, sont arrangés de façon périodique dans un cristal avec des distances proches de $\boldsymbol{\lambda}$. Les ondes diffusées par ces atomes interferent : c'est le phénomène de diffraction (voir glossaire, p. 28). À partir de l'analyse de la diffraction des rayons $\mathrm{X}$, il est donc possible de déduire la façon dont les atomes s'organisent dans l'espace [1]. Ceci permet de comprendre, par exemple, pourquoi un matériau est conducteur, transparent, magnétique... La relation structure-fonction est aussi fondamentale dans les sciences du vivant.

Si le principe général de telles expériences a peu évolué en un siècle, de nouvelles technologies ont vu le jour, permettant une description de plus en plus précise de la structure atomique (voire électronique) de la matière. Tout d'abord au niveau des détecteurs, en passant de plaques photographiques à des détecteurs électroniques plus sensibles. Ensuite, au niveau des moyens de calculs : les ordinateurs toujours plus puissants permettent d'analyser une quantité gigantesque de données. Enfin, au niveau des sources de rayonnement $\mathrm{X}$ de plus en plus brillantes. Les tubes à rayons $\mathrm{X}$, encore utilisés de nos jours dans nos laboratoires, sont des sources divergentes et incohérentes; leur brillance est donc faible. Les synchrotrons, comme l'ESRF à Grenoble ou SOLEIL sur le plateau de Saclay [2], accélèrent des électrons dans un anneau, ce qui génère des rayons $\mathrm{X}$ (c'est le rayonnement synchrotron). La brillance des synchrotrons est très élevée car le flux de photons $\mathrm{X}$ est grand, la taille de la source est petite et le faisceau est peu divergent.

Les lasers à électrons libres dans le domaine des rayons $\mathrm{X}$ (X-FEL pour $X$-ray Free Electron Laser, fig. 1) sont des sources de rayonnement $\mathrm{X}$ sur accélérateur de particules de quatrième génération, qui génèrent un rayonnement laser de brillance phénoménale. Dans la partie I de cet article, nous expliquons comment fonctionne un X-FEL. Nous présentons ensuite dans la partie II quelques applications majeures tirant avantage des impulsions de rayons $\mathrm{X}$ de quelques femtosecondes ( $\left.1 \mathrm{fs}=10^{-15} \mathrm{~s}\right)$, permettant d'étudier la transformation de la matière sur des échelles de temps correspondant à celles des mouvements atomiques élémentaires. La brillance fantastique de ces machines ouvre aussi de nouvelles opportunités pour étudier des nanocristaux. La cohérence de ce rayonnement permet enfin de développer des expériences d'imagerie, avec une résolution spatiale qui pourrait approcher la taille d'un atome. Il est alors envisageable d'étudier la structure d'une protéine ou d'un virus, sans avoir besoin de les faire cristalliser. 

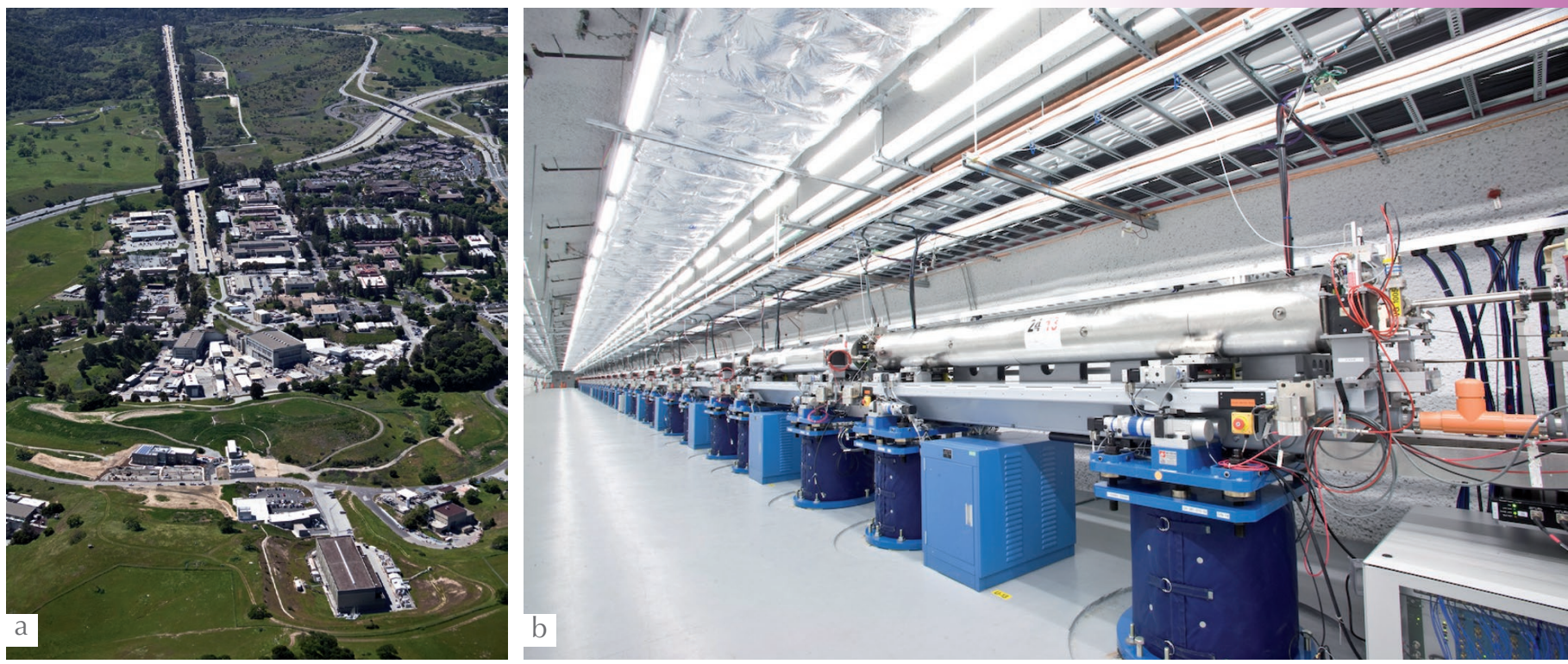

1. L'installation X-FEL LCLS à Stanford (Linac Coherent Light Source, http://Ils.slac.stanford.edu) a été le premier X-FEL, entré en opération en 2009. L'accélérateur (a) et les onduleurs (b) produisant le rayonnement laser se trouvent dans le bâtiment linéaire.

\section{I - Le rayonnement laser à électrons libres dans le domaine X}

Les lasers ont complètement bouleversé le paysage de la recherche et ont investi la vie courante [3]. Le principe du pompage optique fut appliqué par C. Townes et A. Schawlow en 1958 au laser à rubis, puis dans différents milieux amplificateurs comme les lasers à gaz, à semi-conducteur...

C'est en 1971 que J.M.J. Madey à Stanford propose d'utiliser des électrons relativistes dans un champ magnétique alterné (produit par un " onduleur ») comme milieu amplificateur (fig. 2). Un onduleur crée un champ magnétique permanent perpendiculaire à la direction de propagation et périodique selon cette direction. Il peut être fabriqué à partir d'aimants permanents et de pôles magnétiques, ou d'électro-aimants. L'émission spontanée est produite par le rayonnement synchrotron émis par les particules relativistes accélérées par le champ magnétique de l'onduleur. Le rayonnement de l'onduleur est accordable par simple modification de son champ magnétique [2].

Dans le cas du laser à électrons libres (FEL), à la différence du rayonnement synchrotron d'un anneau de stockage, les électrons relativistes dans l'onduleur échangent de l'énergie avec les photons ; certains électrons gagnent alors de l'énergie, d'autres en perdent et, au fur et à mesure qu'ils progressent, ils se retrouvent en avant ou en arrière dans le paquet d'électrons (fig. 2). La modulation en énergie se transforme ainsi en modulation en densité d'électrons à la longueur d'onde du rayonnement émis, les émetteurs sont mis en phase, ce qui leur permet de générer des rayons $\mathrm{X}$ de façon cohérente dans l'onduleur. L'onde lumineuse est alors amplifiée au détriment de l'énergie cinétique des électrons.

Le premier laser à électrons libres rayonna dans l'infrarouge en configuration oscillateur, et fut construit sur un accélérateur linéaire à Stanford en 1977. À l'époque, beaucoup d'espoir avait été mis dans l'emploi d'accélérateurs à recirculation, et une collaboration entre l'équipe de Stanford et l'équipe française qui travaillait sur l'anneau de stockage d'Orsay (ACO) fut à l'origine du second laser à électrons libres au monde. a
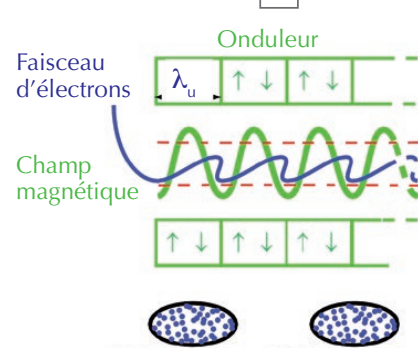

ज्ञ5n-

Émission

Émission Modulation spontanée en énergie
Depuis, le domaine est en pleine expansion, avec de nouvelles configurations. Mais en l'absence de miroirs performants pour la cavité optique dans le domaine des rayons $\mathrm{X}$, le régime d'émission spontanée auto-amplifiée (SASE : Self Amplified Spontaneous Emission) fut proposé en 1980 (fig. 2). L'émission spontanée générée en début d'onduleur est amplifiée en un seul passage au fur et à mesure de la progression du paquet d'électrons. Cette configuration SASE est couramment employée à présent, et un nouveau mode d'injection permet

>>
2. Schéma de laser à électrons libres (FEL) en configuration SASE (émission spontanée auto-amplifiée). (a) Les électrons (points bleus) soumis au champ magnétique (en vert) de l'onduleur ( $\uparrow$ ou $\downarrow$ ) oscillent et émettent du rayonnement synchrotron (ondes rouges), l'émission spontanée du FEL.

(b) Ce rayonnement interagit ensuite petit à petit avec les électrons au fur et à mesure de leur progression dans l'onduleur : un échange d'énergie se produit, certains électrons perdent un peu d'énergie et se trouvent ralentis, d'autres en gagnent se retrouvent en avant du paquet. Le paquet d'électrons devient modulé en densité selon la direction longitudinale, à la longueur d'onde de l'onduleur, et l'émission est alors cohérente. (c) L'onde de lumière est ensuite amplifiée au fur et à mesure de sa progression dans l'onduleur. Enfin, se produit une surmodulation du paquet et un échange cyclique entre absorption et gain, le laser X sature.

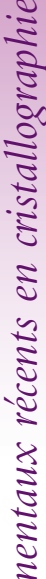




\section{\>}

une amplification plus rapide et un meilleur contrôle des propriétés du rayonnement généré.

Pour rayonner dans le domaine des rayons X, l'énergie des électrons doit être assez élevée (1-20 GeV). Le gain du FEL est d'autant plus grand que la densité électronique est élevée, que l'onduleur est long et que l'énergie des électrons est basse ; pour le domaine $\mathrm{X}$, on emploie donc en général des accélérateurs linéaires performants, permettant de produire des paquets d'électrons courts dans la gamme $10 \mathrm{fs}-10 \mathrm{ps}\left(1 \mathrm{ps}=10^{-12} \mathrm{~s}\right)$, ayant des dimensions très faibles conduisant à une densité électronique élevée, ainsi que des onduleurs très longs ; les installations atteignent alors quelques $\mathrm{km}$. Les X-FELs (sources dites de quatrième génération) se distinguent du rayonnement synchrotron par la production d'impulsions ultra-courtes (femtoseconde) et par la cohérence longitudinale apportée grâce au processus de laser à électrons libres. En conséquence, leurs brillances crêtes et moyennes sont très élevées, de plusieurs ordres de grandeur supérieures à celles des synchrotrons.

Près de 30 ans après le premier FEL et un peu plus de cinquante ans après la découverte du laser, l'avènement des FELs dans le domaine UV-X (Flash 2005, DESY, Hambourg) puis dans le domaine des rayons X avec LCLS (Stanford, USA, en avril 2009, plusieurs mJ à 1,5 $\AA$ [4]) et SACLA (RIKEN, Japon, juin 2011 [5]), permet une moisson de premiers résultats scientifiques spectaculaires.

De nouveaux lasers à électrons libres dans le domaine $\mathrm{X}$ sont en construction en Suisse (Swiss FEL), en Corée (PAL FEL) et en Allemagne (European X-FEL, www.xfel.eu). Ce dernier, construit à Hambourg dans le cadre d'une collaboration européenne, sera le premier X-FEL implanté sur un accélérateur linéaire supraconducteur ; ses performances optimisées faciliteront le développement de ce type d'expérience.

En France, un projet démonstrateur de FEL avancé (LUNEX5) dans la gamme 40-4 nm prépare déjà l'un des possibles successeurs des FELs : une fréquence de répétition élevée, une taille et un coût considérablement réduits via des schémas d'injection, des onduleurs compacts de fort champ, l'emploi d'électrons accélérés par un laser dans un plasma, permettront de développer des nouvelles expériences.
Instantané atomique

encadré 1

À l'instar du photographe E. Muybridge, qui a séquencé par des photos successives le mouvement d'un cheval au galop en 1878, il est possible de suivre des mouvements atomiques à l'aide de techniques pompe-sonde. Une première impulsion laser optique, dite " pompe ", équivalente au coup de talon sur le flanc du cheval, déclenche la transformation du cristal. Une seconde impulsion de rayons $X$, dite "sonde », équivalente à la lumière d'un néon stroboscopique, mesure à un instant $t$ après l'excitation optique la position des atomes en mouvement. En variant le retard $t$ entre ces deux impulsions, il est possible de recomposer le film de l'évolution de la structure atomique du cristal. Les impulsions $X$ femtoseconde générées par les X-FELs permettent d'observer les mouvements des atomes.

Cependant, comme il est nécessaire d'accumuler plusieurs tirs, la résolution temporelle est limitée par le contrôle de la différence $t$ des temps d'arrivée des impulsions optique et X. À cause des limitations technologiques, $t$ fluctue de tir à tir et la précision de la mesure n'est alors que de plusieurs centaines de femtosecondes. Ceci donne une image floue de l'évolution temporelle de la structure atomique (fig. E1d gauche).

Afin de pallier ce problème, il faut alors mesurer pour chaque tir le temps d'arrivée relatif entre impulsions $\mathrm{X}$ et optique. Une solution consiste à convertir l'information temporelle en information spatiale (fig. E1abc). Les deux impulsions arrivent sur une membrane transparente à l'impulsion optique. L'impulsion X arrive sur la membrane avec un angle d'incidence rasant à un instant $t_{1}$, la rendant optiquement opaque (effet d'ionisation intense quasiment ultra-bref, mais transitoire). À l'instant $t_{2}>t_{1}$, l'impulsion laser optique vient sonder la membrane rendue opaque par l'impulsion $X$ sur une partie correspondant au temps $t=t_{2}-t_{1}$. La portion de membrane rendue opaque est imagée sur un détecteur, donnant le temps d'arrivée relatif $t$ des deux impulsions. Sur la figure E1, trois conditions différentes sont représentées : I'impulsion $X$ arrive sur la membrane après (a), en même temps que (b) ou avant (c) l'impulsion optique. Le profil spatial de la transmission de l'échantillon (fig. E1b) donne une mesure directe du décalage temporel entre pompe et sonde. La résolution temporelle ultime atteint quelques fs, donnant alors une image nette de l'évolution temporelle de la structure atomique (fig. E1d droite) et permettant de voir bouger les atomes (fig. $3 \mathrm{~b}$ ).
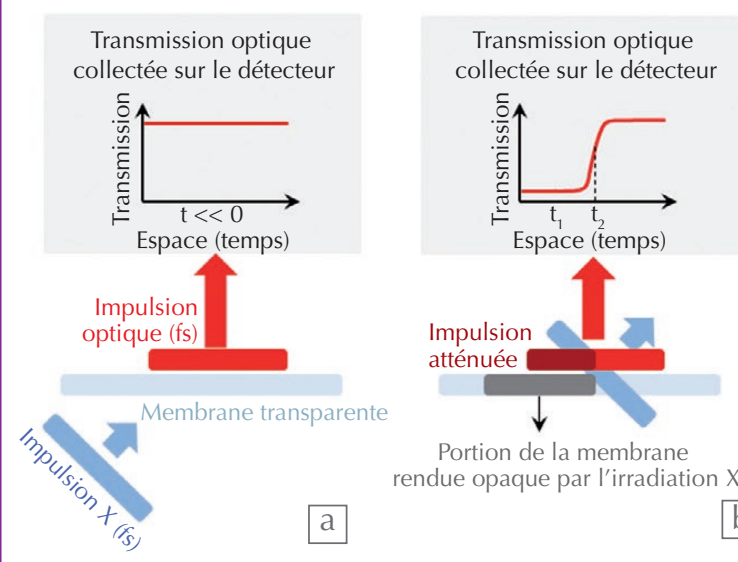

Transmission optique collectée sur le détecteur 气ิ

\section{E1. Principe de la synchronisation temporelle.}

(a) L'impulsion X (en bleu) arrive longtemps après l'impulsion optique (en rouge) sur la membrane transparente (en bleu clair). (b) Les deux impulsions arrivent en même temps, et une partie de la membrane est rendue opaque par l'ionisation X. Ceci se traduit directement par un changement de la transmission optique. La position du changement brutal de transmission correspond au moment $t_{2}$ où les impulsions optique et $X$ sont synchronisées.

(c) L'impulsion optique arrive longtemps après l'impulsion X et la membrane est complètement opaque.

(d) Sans synchronisation temporelle précise, l'information sur la position $x$ des atomes est limitée dans le temps (ellipsoïdes étirés, à gauche). La synchronisation donne des instantanés de la structure cristalline (à droite), avec ici une résolution de 50 fs (et pouvant atteindre $6 \mathrm{fs}$ ). 


\section{II - Exemples d'applications des X-FELs}

\section{Études structurales ultra-rapides : voir bouger les atomes en temps réel}

Un des aspects les plus fascinants de la matière est sa capacité à faire évoluer ses propriétés (magnétisme, conductivité, supraconductivité, couleur...) sous l'effet de contraintes extérieures. Ces propriétés macroscopiques sont liées à la structure microscopique de la matière et à la façon dont ses constituants (électrons, atomes, molécules) s'organisent. Comprendre et contrôler ces réponses ouvre la voie à de nouvelles applications technologiques ou à de nouveaux dispositifs.

En plus de l'intérêt de réduire la taille de ces dispositifs (en particulier autour des nanotechnologies), il est important de pouvoir les contrôler sur des échelles de temps très rapides. L'échelle de temps caractéristique des mouvements atomiques étant de 100 fs (soit 100 millionièmes de milliardième de seconde), on peut espérer comprendre le changement de structure associé au changement de fonction d'un objet sur cette échelle de temps [6]. Les expériences de diffraction des rayons $\mathrm{X}$ résolues en temps, à l'aide d'impulsions $\mathrm{X}$ ultra-brèves (de l'ordre de $10 \mathrm{fs}$ ), permettent à présent d'observer les mouvements atomiques en temps réel et ouvrent une nouvelle ère pour comprendre cette matière dynamique et transformable.

\section{Structure de la matière et diffraction des rayons $\mathbf{X}$}

Pour comprendre le fonctionnement de ces expériences, prenons le cas simple d'un cristal de bismuth $[6,7]$. Ce cristal est constitué d'un motif de deux atomes qui se répète de façon périodique dans l'espace (période normalisée de longueur 1), où distances interatomiques courtes et longues alternent (fig. 3a). Appelons $x$ la distance courte entre deux atomes de bismuth et $f_{\mathrm{Bi}}$ le facteur de diffusion atomique des rayons X par le bismuth. Le facteur de structure, qui décrit la diffusion des rayons X par cette structure cristalline (voir glossaire, p. 28), s'écrit alors : $F_{h k l}=2 f_{\mathrm{Bi}} \cos [\pi(h+k+l) x]$ (1) et, pour un faisceau de rayons $\mathrm{X}$ diffracté suivant la direction $(h=k=l=1)$, l'intensité mesurée est simplement :

$$
I_{111}(t)=4 f_{B i}^{2} \cos ^{2}[3 \pi \mathrm{x}](2) .
$$

Lorsque le cristal est à l'équilibre, la distance $x=x_{0}$ est définie par la façon dont les électrons, qui font la colle entre les noyaux, se répartissent sur des niveaux d'énergie plus ou moins liants, donnant ainsi un potentiel périodique $E_{0}(x)$ (fig. $3 a$ ). C'est en mesurant les intensités diffractées suivant des directions $\left(\begin{array}{lll}h & k\end{array}\right)$ que l'on observe qu'à l'équilibre $x=x_{0}=0,467$.

\section{La lumière déplace les atomes}

Si on excite ce cristal à l'aide d'une impulsion lumineuse laser ultra-brève (10 fs), l'énergie contenue dans cette impulsion est transférée aux électrons qui vont occuper d'autres niveaux d'énergie moins liants. Ceci donne un nouveau potentiel $E_{1}(\mathrm{x})$, modifié de façon quasiinstantanée (à un instant référence $t=0$ ) par l'impulsion lumineuse, associé à une nouvelle distance d'équilibre $x_{1}$. Le potentiel $E_{1}(x)$ étant périodique, cette modification concerne tous les atomes



du cristal, qui doivent alors partir de façon synchrone d'une position d'équilibre initiale $x_{0}$ vers leur nouvelle position d'équilibre $x=x_{1}$.

La figure $3 \mathrm{~b}$ montre l'évolution en temps réel de l'intensité diffractée $I_{111}(\mathrm{t})$ mesurée après excitation laser [7]. Réussir un tel exploit n'est possible qu'avec une résolution temporelle meilleure que le temps caractéristique des mouvements atomiques, ce qui nécessite une parfaite synchronisation entre le laser optique qui excite le cristal et le laser à rayons X qui vient mesurer sa structure à un instant donné (voir l'encadré, p. 46). L'équation (2) relie l'intensité des rayons $\mathrm{X}$ diffractée sur la raie de Bragg (111) à la distance $x_{\mathrm{Bi}-\mathrm{Bi}}$. On déduit directement de l'évolution temporelle de $I_{111}(\mathrm{t})$ celle de $x(\mathrm{t})$ (fig. 3b). La trajectoire décrite par les atomes est celle d'un oscillateur amorti : $x(t)=x_{1}+\left(x_{0}-x_{1}\right) \cos (2 \pi f t) \exp (-t / \tau)$.

3. Évolution structurale du bismuth après une excitation laser.

(a) Haut : structure du bismuth dans l'état stable, où le potentiel $E_{0}(x)$ donne une distance d'équilibre $\mathrm{x}=\mathrm{x}_{0}$ entre les atomes de Bi en vert. Cette structure se répète de façon périodique dans le cristal (période normalisée à 1). Bas : structure du bismuth dans l'état photo-excité. Le potentiel $\mathrm{E}_{1}(\mathrm{x})$ créé instantanément par la lumière donne une nouvelle distance d'équilibre $x=x_{1}$. Les atomes se déplacent et oscillent alors autour de cette nouvelle position d'équilibre (trajectoire en violet).

(b) À partir de la mesure de la variation relative de l'intensité des rayons $X$ diffractés en fonction du temps $\left(\left.\right|_{111}(t)\right.$, axe de gauche), on déduit l'évolution de la distance Bi-Bi en fonction du temps ( $x(t)$, axe de droite). 
\〉

Cette trajectoire dans le nouveau potentiel d'une position initiale $x_{0}$ vers une position finale $x_{1}$ est représentée de façon schématique en violet sur la figure $3 \mathrm{a}$. On déduit de ces mesures la fréquence $f$ de vibration de la paire d'atomes Bi-Bi (ici de $\sim 2,7 \mathrm{THz}$ ) dans le potentiel de l'état photo-excité, ainsi que le déplacement relatif des atomes de Bi correspondant à l'amplitude de l'oscillation de 0,016 Å. Ceci souligne l'extrême sensibilité des techniques de diffraction à la façon dont les atomes s'organisent dans l'espace. On déduit aussi de cette observation la façon dont la nouvelle position d'équilibre est atteinte, avec une constante d'amortissement $\tau \cong 1$ ps. Ces observations directes permettent de modéliser la dynamique hors équilibre liée à ces effets photo-induits et de mieux décrire le couplage entre les degrés de liberté électroniques et structuraux de la matière (équilibre des températures des soussystèmes que sont les électrons et le réseau cristallin). Cet exemple "simple" démontre la capacité des expériences résolues en temps à suivre directement la réorganisation de la matière aux échelles atomiques de temps et d'espace.

Ces études ultrarapides se développent en physique, chimie et biologie. Elles permettent de suivre en temps réel le chemin de transformation pour comprendre l'émergence de fonctions induites par la lumière [6].

\section{Transformation de biomolécules par la lumière}

Ce qui est facilement descriptible pour un cristal à deux atomes par maille peut être développé pour des systèmes plus complexes comme des molécules ou des biomolécules. À titre d'exemple, on peut mentionner l'étude sur la protéine jaune photo-active (PYP) [8], qui est le photorécepteur de la bactérie Halorhodospira halophila. L'absorption d'un photon visible déclenche un cycle photochimique complexe, où différentes espèces intermédiaires se forment dans un intervalle de temps compris entre 100 fs et plusieurs secondes. Les études de cristallographie ultrarapide (fig. 4) ont permis d'observer l'isomérisation du chromophore (groupe fonctionnel qui apporte la couleur), c'est-à-dire les mouvements des atomes liés au changement de la structure de la protéine.

\section{Cristallographie en série : tueur en série de cristaux}

À capacité maximale, la brillance des X-FEL est telle qu'elle permet d'étudier des cristaux de taille nanométrique. Mais dans ces conditions extrêmes, le rayonnement X-FEL ionise la matière. Ceci est intéressant pour la physique atomique et a permis par exemple d'ioniser fortement des atomes relativement lourds comme le xénon. Mais c'est un problème limitant pour la cristallographie, puisque les cristaux peuvent être détruits. Heureusement, le temps d'interaction entre ces impulsions $\mathrm{X}$ extrêmement brèves et la matière est suffisamment court pour que le phénomène de diffraction se produise avant que la matière ne soit détruite [9]. Cependant, les études de diffraction des rayons X nécessitent de sonder le cristal dans différentes directions pour connaitre en trois dimensions la position des atomes quile composent. Habituellement, les données de diffraction $\mathrm{X}$ sont collectées sur un même cristal pour différentes orientations. Ces mesures répétées sont


a donc problématiques dans les cas où le faisceau de rayons $\mathrm{X}$ dégrade (ou détruit!) le cristal.

Pour pallier ces limitations, les chercheurs tirent avantage de la brillance ultime des X-FELs en repensant complètement la façon de réaliser des mesures (fig. 5) avec une nouvelle méthode connue sous le nom de "Serial Femtosecond Crystallography" (cristallographie femtoseconde en série) [9]. Cette méthode utilise un réservoir de cristaux (de taille $100 \mathrm{~nm}$ à $10 \mu \mathrm{m}$ ) en suspension, qu'un injecteur sophistiqué place un à un dans le faisceau de rayons $\mathrm{X}$ environ cent fois par seconde. Le signal de diffraction est ainsi collecté dès qu'une impulsion de rayons $\mathrm{X}$ frappe un cristal. Contrairement aux méthodes conventionnelles, ce cristal n'est utilisé ici qu'une fois car il est détruit (ou détérioré) après avoir été exposé aux rayons X. Des chercheurs ont démontré le principe de fonctionnement de ce concept. Des projets encore plus ambitieux se développent actuellement, faisant du X-FEL un véritable tueur de cristaux en série (des millions de cristaux sont utilisés par expérience). b

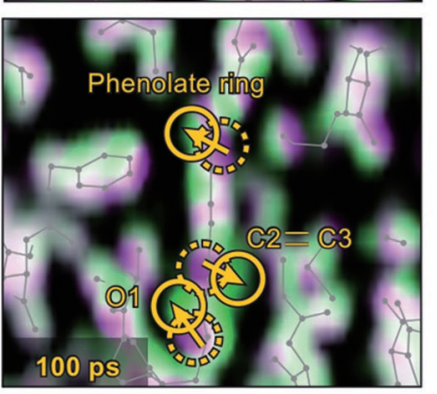

4. Changements structuraux observés dans la protéine PYP entre son état initial et 100 ps après une excitation lumineuse.

(a) Dans l'état initial (à gauche), les images montrent la répartition de la densité électronique (en blanc), déduite du spectre de diffraction X-FEL. L'arrangement moléculaire est indiqué schématiquement.

(b) Dans l'état excité (à droite), l'isomérisation du chromophore, liée aux mouvements moléculaires indiqués par les flèches, est montrée ici dans la première étape de la transformation. Les couleurs indiquent les déplacements des atomes de la protéine de leurs positions initiales (en violet) vers leurs nouvelles positions (en vert). Ce n'est que le début d'une relaxation complexe de la protéine, s'étalant sur plusieurs décades temporelles. [Reprinted with permission from Macmillan Publishers Ltd: Nature Chemistry [8], copyright 2013.] 


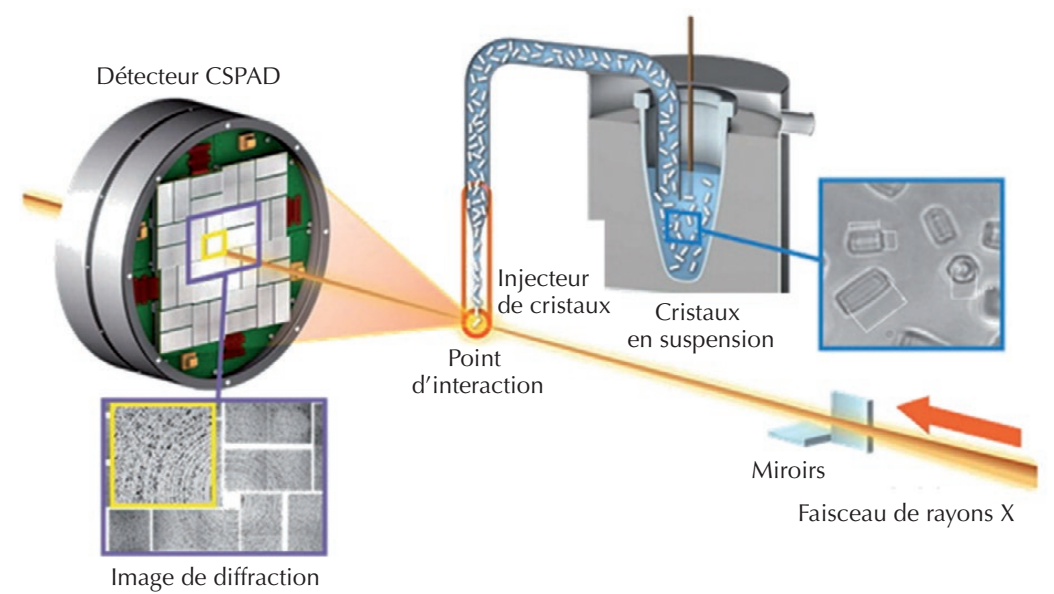

5. Principe de la cristallographie femtoseconde X en série [9]. Des cristaux en suspension dans un réservoir sont injectés un à un dans le faisceau de rayons $X$ (en orange) pulsé. Un premier cristal diffracte les rayons $X$, collectés sur le détecteur, puis est remplacé par un nouveau cristal qui va à son tour diffracter les rayons $X$, etc. On obtient ainsi le diagramme de diffraction. [Modified from [9]. Reprinted with permission from AAAS.]

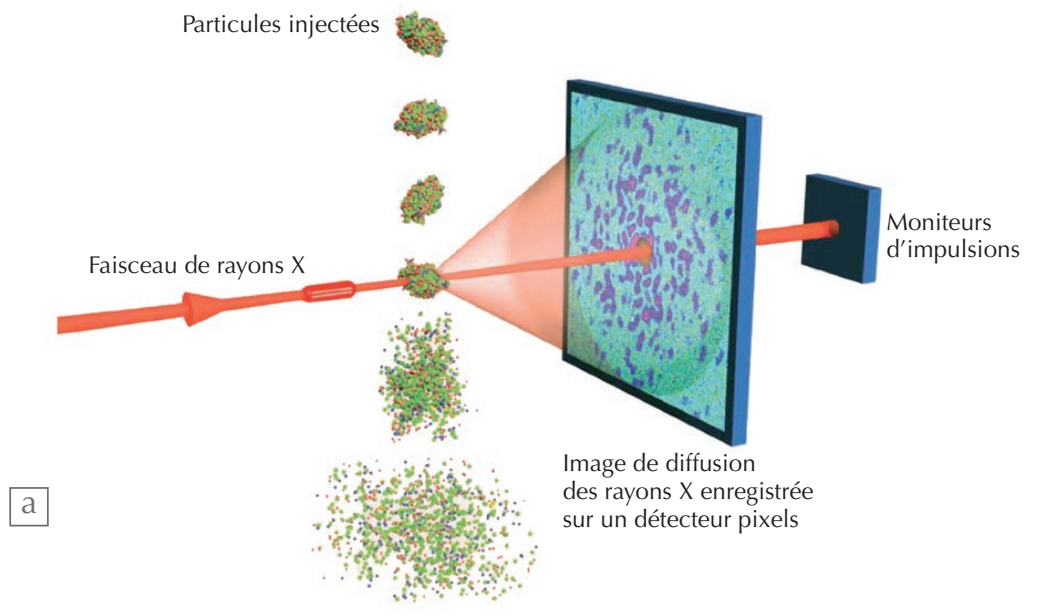

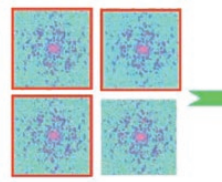

Classification



Moyenne

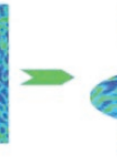

Orientation

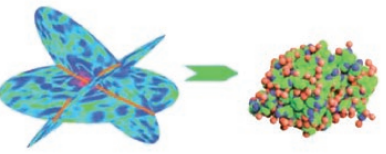

Reconstruction
6. Représentation schématique du principe d'imagerie par diffusion cohérente des rayons X par une particule unique.

(a) Le faisceau de rayons X est diffusé par la particule orientée de façon aléatoire et l'image est enregistrée sur un détecteur. Ce faisceau détruit la particule et on ne peut obtenir qu'une vue $2 \mathrm{D}$ pour chaque image. De nouvelles images sont collectées pour chaque particule qui traverse le faisceau de rayons $X$.

(b) Ces très nombreuses données sont ensuite classifiées et regroupées pour différentes orientations des particules, puis moyennées. Les données de diffusion des rayons $X$ sont alors reconstruites en $3 D$, en tenant compte de ces orientations. L'image de la particule est ensuite obtenue par récupération de phase. [From [10]. Reprinted with permission from AAAS.]

\section{Références}

1 A. Authier, « Une découverte qui a changé le monde: la diffraction des rayons X», Reflets de la physique 39 (2014) 24.

2• Reflets de la physique 34-35 (juin 2013), « La lumière synchrotron, au service de la science et de la société ».

3- Reflet de la physique 21 (octobre 2010), « Le laser : 50 ans de découvertes".

4 P. Emma et al., Nature Photonics 4 (2010) 641.
5. Ishikawa et al." "A compact X-ray free-electron Photonics 6 (2012) 540-544.

6• É. Collet, "Dynamical structural science", Acta Cryst. A 66 (2010) 133-134.

7• M. Harmand et al., "Achieving few-femtosecond time-sorting at hard X-ray free-electron lasers", Nature Photonics, 7 (2013) 215-218.

\section{Cristallographie sans cristaux : vers l'imagerie à l'échelle atomique}

Le principe de la diffraction des rayons $\mathrm{X}$, basé sur les interférences créées par des (bio)molécules arrangées de façon périodique dans les cristaux, trouve ses limites lorsque l'on s'intéresse à des systèmes qui ne peuvent former de cristaux. De plus, certaines fonctions nécessitant des changements de structure importants peuvent être inhibées dans les cristaux. Il a alors été suggéré d'étudier le rayonnement $\mathrm{X}$ diffusé par des molécules isolées, suivant le principe mentionné ci-dessus pour la cristallographie en série. Des centaines de milliers d'images de diffusion des rayons $\mathrm{X}$ par un type d'objet (une macromolécule, une protéine, un virus...) sont collectées, moyennées puis orientées pour obtenir une image 3D de l'objet dans l'espace des vecteurs d'onde (fig. 6). Il est alors possible à partir de cette «transformée de Fourier» de l'objet de remonter à sa structure dans l'espace réel 3D en utilisant un algorithme d'inversion itératif [10]. De telles études semblent très difficiles. Ce n'est que très récemment qu'a été montrée la possibilité d'imager la structure d'un virus, bien que ceci ne soit encore réalisé qu'à deux dimensions et avec une résolution spatiale limitée. Le rêve d'étudier la matière (et des macromolécules) sans se soucier de les faire cristalliser semble à notre portée, mais il faudra encore attendre de nombreuses années avant d'atteindre une résolution spatiale à l'échelle atomique.

\section{Conclusion}

Les X-FELs sont donc au cœur d'une révolution scientifique. De nouvelles expériences, en particulier en lien avec la cristallographie, tirent profit des capacités exceptionnelles de ces machines afin d'appréhender les propriétés structurales et dynamiques de la matière. De nouveaux champs d'exploration s'ouvrent alors autour d'une physique liée à des conditions extrêmes de temps, d'espace ou d'énergie. I

8• Y. Jung et al., Nature Chemistry, 5 (2013) 212-220.

9- S. Boutet et al., "High-Resolution Protein Structure Determination by Serial Femtosecond Crystallography", Science 337 (2012) 362.

10• K. J. Gaffney et H. N. Chapman, "Imaging Atomic Structure and Dynamics with Ultrafast X-ray Scattering", Science 316 (2007) 1444-1448. 